\title{
Neurologische Erkrankungen und Schwangerschaft - eine Übersicht
}

\author{
Neurological Disorders and Pregnancy
}

P. Berlit

Neurologische Klinik, Alfried Krupp Krankenhaus, Essen

\section{Zusammenfassung}

$\nabla$

Neurologische Erkrankungen, die durch eine Schwangerschaft ausgelöst werden, sind das posteriore reversible Enzephalopathiesyndrom (PRES), das Fruchtwasser-Embolie-Syndrom, das Sheehan-Syndrom und die Post-partum-Angiopathie - das reversible zerebrale Vasokonstriktionssyndrom (RCVS). Zu den diagnostischen Kriterien der Präeklampsie zählen Blutdruckerhöhung und Proteinurie; bei epileptischen Anfällen liegt eine Eklampsie vor. Hämolyse, erhöhte Leberenzyme und niedrige Thrombozytenzahlen (low platelets) sind Leitsymptome des HELLP-Syndroms. Sehstörungen bis zur kortikalen Blindheit charakterisieren das posteriore reversible Enzephalopathiesyndrom (PRES). Das Sheehan-Syndrom zeigt sich als Hypopituitarismus post partum und kommt durch einen Apoplex der Hypophyse bei peripartalen Blutungen mit längerfristigem Blutdruckabfall zustande. $\mathrm{Zu}$ den neurologischen Krankheiten, die während einer Schwangerschaft gehäuft auftreten, zählen Schlaganfälle, die Sinusvenenthrombose, das Restless-Legs-Syndrom und Nervenkompressionssyndrome. Chronische neurologische Erkrankungen müssen während einer Schwangerschaft interdisziplinär überwacht und betreut werden. Die Einnahme von Antikonvulsiva während der Schwangerschaft verdoppelt das Risiko von Geburtsdefekten; das höchste Risiko besteht für Valproinsäure. Das geringste Fehlbildungsrisiko weisen Lamotrigin und Levetiracetam auf. Bei MSPatientinnen sind nach Registerdaten Glatirameracetat und Interferone als Intervalltherapeutika während der Gravidität relativ sicher. Alle anderen Substanzen sollten abgesetzt werden.

\section{Abstract \\ $\nabla$}

Neurological disorders caused by pregnancy and puerperium include the posterior reversible encephalopathy syndrome, the amniotic fluid embolism syndrome (AFES), the postpartum angiopathy due to reversible vasoconstriction syndrome, and the Sheehan syndrome. Hypertension and proteinuria are the hallmarks of preeclampsia, seizures define eclampsia. Hemolysis, elevated liver enzymes and low platelets constitute the HELLP syndrome. Vision disturbances including cortical blindness occur in the posterior reversible encephalopathy syndrome (PRES). The Sheehan syndrome presents with panhypopituitarism post partum due to apoplexia of the pituitary gland in severe peripartal blood loss leading to longstanding hypotension. Some neurological disorders occur during pregnancy and puerperium with an increased frequency. These include stroke, sinus thrombosis, the restless legs syndrome and peripheral nerve syndromes, especially the carpal tunnel syndrome. Chronic neurologic diseases need an interdisciplinary approach during pregnancy. Some anticonvulsants double the risk of birth defects. The highest risk exists for valproic acid, the lowest for lamotrigine and levetiracetam. For MS interval treatment, glatiramer acetate and interferones seem to be safe during pregnancy. All other drugs should be avoided.
VNR

2760512016149751616

\section{Bibliografie}

Dol http://dx.doi.org/ 10.1055/s-0041-108911

Fortschr Neurol Psychiatr 2016; 84: 103-113 @ Georg Thieme Verlag KG Stuttgart . New York . ISSN 0720-4299

Korrespondenzadresse Prof. Dr. med. Peter Berlit Neurologische Klinik, Alfried Krupp Krankenhaus Alfried-Krupp-Straße 21 45131 Essen peter.berlit@KruppKrankenhaus.de 


\section{Lernziele}

$\nabla$

- Diagnose und Therapie von Erkrankungen, die durch eine Schwangerschaft ausgelöst werden: Präeklampsie, Eklampsie, HELLP-Syndrom, Fruchtwasser-Embolie-Syndrom, SheehanSyndrom und Post-partum-Angiopathie.

- Kenntnis neurologischer Erkrankungen mit erhöhter Häufigkeit während einer Schwangerschaft: Schlaganfälle, Restless-Legs-Syndrom Nervenkompressionssyndrome.

- Diagnostik und Therapie einer Sinusvenenthrombose während einer Schwangerschaft und im Wochenbett.

- Management von chronischen neurologischen Erkrankungen während einer Schwangerschaft (Migräne, Multiple Sklerose, Myasthenia gravis, Epilepsie).

\section{Einleitung \\ $\nabla$}

Neurologische Erkrankungen können bereits vor einer Schwangerschaft bestehen oder sich erstmals während einer Schwangerschaft oder im Wochenbett manifestieren. In beiden Fällen ist eine sachkundige interdisziplinäre Beratung und Betreuung der betroffenen Frauen durch Gynäkologen und Neurologen notwendig. Dieser klinisch wichtigen Thematik widmet sich diese Übersichtsarbeit.

Zu den Erkrankungen, die durch eine Schwangerschaft ausgelöst werden können, zählen neben Präeklampsie, Eklampsie und HELLP-Syndrom das posteriore reversible Enzephalopathie-Syndrom (PRES), das Fruchtwasser-Embolie-Syndrom, das Sheehan-Syndrom und die Post-partum-Angiopathie - das reversible zerebrale Vasokonstriktionssyndrom (RCVS).

Andere Erkrankungen treten während einer Schwangerschaft mit erhöhter Häufigkeit auf. Dazu zählen Schlaganfälle, die Sinusvenenthrombose im Wochenbett, das Restless-Legs-Syndrom oder Nervenkompressionssyndrome.

Chronische neurologische Erkrankungen, die bereits vor der Schwangerschaft bestanden haben oder sich erstmals während einer Schwangerschaft manifestieren (wie Migräne, Multiple Sklerose, Myasthenia gravis oder Epilepsie), bedürfen der besonders intensiven interdisziplinären $\mathrm{Ab}$ klärung, Überwachung und Betreuung.

Schwangerschaftserkrankungen mit neurologischen Symptomen - Präeklampsie, Eklampsie und HELLP-Syndrom

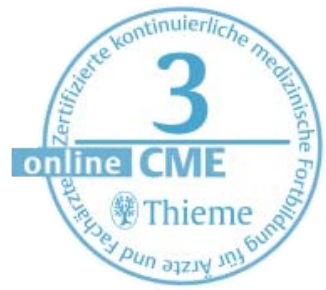

Von einer Präeklampsie wird gesprochen, wenn es im 2. oder 3. Trimenon der Schwangerschaft zu einer dauerhaften Blutdruckerhöhung ( $>140$ / $90 \mathrm{mmHg}$ ) und Proteinurie kommt [1]. Auch wenn Ödeme bei Patientinnen mit Präeklampsie häufig auftreten, zählen sie nicht zu den erforderlichen diagnostischen Kriterien für diese Di- agnose. Wenn es im Rahmen einer Präeklampsie $\mathrm{zu}$ anhaltenden Kopfschmerzen, psychischen Auffälligkeiten und Sehstörungen kommt, wird von einer schweren Präeklampsie gesprochen. Sobald epileptische Anfälle auftreten, liegt eine Eklampsie vor. Für die Entwicklung einer Präeklampsie ist die Placenta Voraussetzung. Wenn es dort zu einer Minderperfusion kommt, resultiert eine systemische endotheliale Dysfunktion aufgrund humoraler angiogener und antiangiogener Faktoren. In der Regel ist die Entbindung kurativ; jedoch kann eine Post-partum-Eklampsie bis zu 6 Wochen nach der Geburt auftreten, ggf. in Assoziation mit einer Post-partum-Angiopathie als Ausdruck eines reversiblen zerebralen Vasokonstriktionssyndroms (RCVS, siehe unten).

Eine Präeklampsie liegt bei dauerhafter Blutdruckerhöhung (>140/90 mmHg) und Proteinurie im 2. oder 3. Trimenon der Schwangerschaft, eine Eklampsie bei zusätzlichen epileptischen Anfällen vor.

Sehstörungen bei einer Eklampsie können so ausgeprägt sein, dass es zur kortikalen Blindheit kommt. In der Regel sind aber auch massive Gesichtsfeldausfälle reversibel im Sinne eines posterioren reversiblen Enzephalopathiesyndroms (PRES), entscheidend ist die zügige Kontrolle von Hypertonie und einer etwaigen Nephropathie. Das PRES stellt das pathophysiologische Korrelat der Eklampsie dar mit initialem vasogenem Ödem ohne Diffusionsstörung. Die im Verlauf in der Magnetresonanztomografie (MRT) nachweisbaren okzipitalen Veränderungen mit Diffusionsstörung und Kontrastmittelaufnahme sind dabei grundsätzlich reversibel [2].

Das HELLP-Syndrom ist eine besonders schwere Form der Präeklampsie mit hoher mütterlicher Morbidität. Leitsymptome sind die Hämolyse, die erhöhten Leberenzyme und die niedrigen Thrombozytenzahlen (low platelets). Beim HELLP-Syndrom treten Kopfschmerzen in bis zu $60 \%$ der Fälle auf, Sehstörungen bei jeder 5. Patientin. Es kann zum Auftreten eines PRES, von Schlaganfällen sowie zur Manifestation einer Eklampsie mit epileptischen Anfällen kommen [3].

Leitsymptome des HELLP-Syndroms sind Hämolyse, erhöhte Leberenzyme und niedrige Thrombozytenzahlen.

Die Therapie der Anfälle bei Eklampsie erfolgt nicht mit Antikonvulsiva, sondern mit Magnesiumsulfat. Nur bei Versagen werden Benzodiazepine hinzugegeben. Eine antikonvulsive Dauertherapie ist nicht erforderlich. 
Bei Vorliegen einer Eklampsie wird Magnesiumsulfat zur Prävention epileptischer Anfälle in der 24. bis 32. Schwangerschaftswoche empfohlen. Bis zur Entbindung muss eine konsequente antihypertensive Behandlung durchgeführt werden [4].

Entscheidende Maßnahme beim HELLP-Syndrom ist die zügige Entbindung, die ab der 34. Schwangerschaftswoche generell empfohlen wird. Falls eine Entbindung zu einem früheren Zeitraum erforderlich ist, sollten vor der Sectio Kortikosteroide gegeben werden, um die fetale pulmonale Entwicklung zu fördern $[5,6]$.

Da Präeklampsie und HELLP-Syndrom nicht vor der 20. Schwangerschaftswoche auftreten, muss bei früherer Manifestation einer Thrombozytopenie mit Thromboseneigung und Schlaganfällen an die thrombotische thrombozytopenische Purpura (TTP) gedacht werden. Die TTP tritt zu $12 \%$ im ersten Trimenon und zu 56\% im zweiten Trimenon der Schwangerschaft auf; im Unterschied zum HELLP-Syndrom sind die Leberwerte nicht verändert bei Vorliegen von Thrombozytopenie, schwerer Anämie und erhöhten LDH-Werten. Bei der TTP kann es auch zu febrilen Temperaturen kommen. Laborchemisch sind vermehrt große Multimere und Fragmentozyten nachweisbar, typisch ist der Nachweis des Von-Willebrand-Faktors.

Eine Thrombozytopenie mit Thromboseneigung und Schlaganfällen im 1. oder 2. Trimenon spricht für das Vorliegen einer thrombotischen thrombozytopenischen Purpura.

\section{Reversibles zerebrales Vasokonstriktions-} syndrom (RCVS)

Leitsymptome des RCVS sind in über $90 \%$ aller Fälle heftige Kopfschmerzen im Sinne von „thunderclap headaches“, die in ihrer Intensität an den Kopfschmerz einer Subarachnoidalblutung (SAB) erinnern. Zusätzlich können neurologische Herdsymptome infolge zerebraler Ischämien oder umschriebener Einblutungen auftreten. Die kraniale MRT zeigt häufig umschriebene Grenzzoneninfarkte. Neben kleinen intrazerebralen Blutungen kommen vor allem sulkale Subarachnoidalblutungen vor. Duplexsonografie, CT- oder MRAngiografie zeigen multilokuläre Gefäßspasmen mit Einschnürungen und Flusserhöhungen. Diese Gefäßveränderungen sind typischerweise innerhalb von 12 Wochen vollständig reversibel [79]. Leider gibt es insbesondere im Wochenbett auch foudroyante und fatale Verläufe [10].

Bei Vorliegen einer umschriebenen SAB wird eine konventionelle digitale Subtraktionsangiografie empfohlen, um eine ursächliche Aneurysmaruptur auszuschließen.

Der Liquor ist in aller Regel normal mit einer Zell-

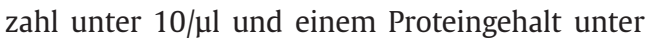
$80 \mathrm{mg} / \mathrm{dl}$.
Schlaganfälle infolge einer „postpartalen Angiopathie“ sind Ausdruck eines RCVS [11]. Das Vasokonstriktionssyndrom manifestiert sich aber nicht nur im frühen Puerperium, sondern auch in der späten Schwangerschaft, vor allem dann, wenn eine Präeklampsie oder ein HELLP-Syndrom vorliegen. Medikamente, die begünstigend wirken können, sind Ergotderivate, Bromocriptin, selektive Serotoninwiederaufnahmehemmer (SSRI), Triptane, Erythrozytenkonzentrate und abschwellende Nasensprays [12].

Therapeutisch werden zerebral wirksame Kalziumantagonisten wie Nimodipin empfohlen, wobei es für diese Therapie keine kontrollierten Studien gibt [13]. Entscheidend ist es, auslösende Faktoren auszuschalten. Sofern dies vertretbar ist, sollte in der späten Schwangerschaft eine zügige Entbindung angestrebt werden. Auf jeden Fall müssen Medikamente, die die Vasokonstriktion verstärken können, strikt vermieden werden.

Das reversible zerebrale Vasokonstriktionssyndrom zeigt sich mit heftigen Kopfschmerzen und oft neurologischen Herdsymptomen durch Ischämien oder Blutungen; die ursächlichen Gefäßspasmen sind innerhalb von 12 Wochen vollständig reversibel. Auftreten als „postpartale Angiopathie“ oder in der späten Schwangerschaft. Therapie mit Nimodipin.

Fruchtwasser-Embolie-Syndrom (Amniotic fluid embolism syndrome - AFES)

Die Inzidenz des AFES wird mit 1 bis 12 Fällen auf 100000 Entbindungen beziffert. Das Risiko ist erhöht bei hohem Lebensalter der Mutter, Multipara (>4 Entbindungen), Kaiserschnitt, Placenta praevia, fetalem Distress-Syndrom, Eklampsie und medikamentöser Einleitung der Geburt.

Das Fruchtwasser gelangt venös über die Plazentaansatzstelle oder Uterusläsionen in die maternale Zirkulation und kann zu kardiogenem Schock, Ateminsuffizienz und Anaphylaxie führen. Leitsymptome sind während der Entbindung oder direkt post partum ein Blutdruckabfall, Sauerstoffmangel mit Atemnot, disseminierte intravasale Gerinnung, Koma und epileptische Anfälle. Entscheidend ist die rasche intensivmedizinische Behandlung der Mutter, um hypoxische Hirnschäden zu verhindern [14].

\section{Apoplex der Hypophyse (Sheehan- Syndrom)}

Der Infarkt der Hypophyse führt zu einem Hypopituitarismus post partum. Es handelt sich um eine potenziell lebensgefährliche Komplikation, die sich allerdings in aller Regel nicht durch neurologische Symptome, sondern durch endokrine Auffälligkeiten zeigt.

Während der Schwangerschaft ist die Hypophyse vergrößert und damit vermehrt anfällig für Ischä-

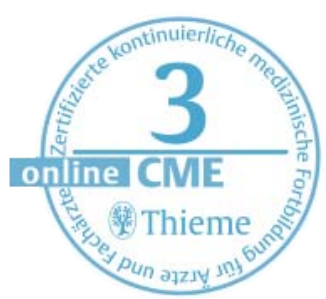


mien, die vor allem durch eine Hypovolämie provoziert werden können. Wenn es unter der Geburt zu transfusionspflichtigen Blutungen mit längerfristigem Blutdruckabfall kommt, kann sich das Sheehan-Syndrom manifestieren. Es gilt an diese Komplikation zu denken, wenn die junge Mutter nicht stillen kann, eine Amenorrhoe post partum vorliegt oder eine anhaltende Hypotension trotz Blutungskontrolle und Volumensubstitution bestehen bleibt. Hinzu kommen oft eine allgemeine Müdigkeit und Anorexie, nur selten zeigt sich ein Diabetes insipidus.

In der Akutphase können Kopfschmerzen und Sehstörungen auftreten. MR-tomografisch lässt sich die vergrößerte ischämisch geschädigte Hypophyse mit Kontrastmittelaufnahme nachweisen; im Verlauf resultiert häufig ein Empty-SellaSyndrom. Der Panhypopituitarismus wird durch Bestimmung von Wachstumshormon, TSH, ACTH, Prolaktin und gonatropen Hormonen belegt. Entscheidend ist die umgehende Hormonsubstitution, um lebensgefährliche Komplikationen zu verhindern $[15,16]$.

Beim Sheehan-Syndrom handelt es sich um den Apoplex der Hyophyse durch Hypovolämie und Blutdruckabfall bei peripartaler schwerer Blutung; Leitsymptom ist der Hypopituitarismus mit Amenorrhoe und Stillunfähigkeit post partum.

Neurologische Erkrankungen, die gehäuft in Verbindung mit einer Schwangerschaft auftreten

Sinus- und Venenthrombose (SVT)

Die SVT hat eine jährliche Inzidenz von 1 auf 100000 Einwohner, wobei Frauen vor dem 40. Lebensjahr bevorzugt betroffen sind. Besonders häufig manifestieren sich SVT während der Schwangerschaft, im Wochenbett oder unter der Einnahme von Ovulationshemmern [17].

Leitsymptome sind subakut auftretende Kopfschmerzen, fluktuierende Herdsymptome, häufig in Form von Monoparesen, sowie bei jeder zweiten Patientin symptomatische epileptische Anfälle oder psychische Auffälligkeiten. Möglich ist auch die Manifestation unter dem Bild eines Pseudotumor cerebri mit bilateraler Stauungspapille, Hirndrucksymptomen und ggf. Abduzensparese. Bei der Sinus-cavernosus-Thrombose verbindet sich eine Protrusio bulbi mit regionaler Schwellung und Ausfällen der Hirnnerven III bis VI.

Neben der Gravidität per se können hereditäre oder erworbene Koagulopathien eine Rolle spielen: Dazu zählen neben der Faktor-V-Leiden-Mutation mit APC-Resistenz die Prothrombinmutation, der AT-III-, Protein-C- oder Protein-S-Mangel, Dysfibrinogenämien, der Plasminogenmangel und das Antiphospholipid-Syndrom (Lupus-Antikoagulans und Kardiolipin-IgG-Antikörper) [18, 19].
Betroffen sind bei einer SVT die Sinus transversus, sigmoideus oder sagittalis superior, seltener die inneren Hirnvenen. Untersuchungsmethode der Wahl ist die kraniale Magnetresonanztomografie mit Phasenkontrast-Angiografie. Die Parenchymbilder zeigen oft zerebrale Ödeme und parasagittale Einblutungen. In der Phasenkontrast-Angiografie kann die Thrombose selbst visualisiert werden. Eine Erhöhung der D-Dimere (> $500 \mathrm{ng} /$ $\mathrm{ml}$ in der Akutphase) ist bei der SVT häufig, kann aber in der Schwangerschaft nur bedingt verwertet werden.

Grundsätzlich ist die Diagnosestellung auch mittels Computertomografie und CT-Angiografie möglich; typisch ist die dreieckförmige Aussparung des Confluens sinuum in der kontrastmittelgestützten CT (empty triangle sign, Deltazeichen) - diese Untersuchungsmethode verbietet sich aber natürlich während der Schwangerschaft aufgrund der Strahlenbelastung.

Entscheidende therapeutische Maßnahme ist die umgehende Antikoagulation mit niedermolekularem Heparin, um ein Fortschreiten der Thrombose zu verhindern. Dies gilt auch bei Nachweis von Diapedeseblutungen in der MRT. Nach der Akutphase erfolgt für 6-12 Monate die orale Antikoagulation mit einem Vitamin-K-Antagonisten und einer Ziel-INR von 2,5 bis 3. Eine über diesen Zeitraum hinausgehende Antikoagulation ist nur bei Vorliegen von Gerinnungsstörungen indiziert [20]. Kommt es trotz effektiver Antikoagulation zu einer Progredienz der Thrombose, kommen als individueller Heilversuch die venöse Thrombektomie oder eine Dekompressionsoperation infrage. Epileptische Anfälle werden in der akuten Phase symptomatisch mit Levetiracetam behandelt. Valproinsäure sollte während der Schwangerschaft nicht eingesetzt werden.

Eine Sinus- und Venenthrombose tritt während der Schwangerschaft oder im Wochenbett auf; Leitsymptome sind Kopfschmerzen, fluktuierende Herdsymptome, und epileptische Anfälle. Diagnose mittels MRT mit Phasenkontrast-Angiografie; Therapie der Wahl ist die Antikoagulation.

\section{Schwangerschaft und Schlaganfall}

Insbesondere junge Frauen haben in Verbindung mit einer Schwangerschaft ein erhöhtes Risiko, einen Schlaganfall zu erleiden [21, 22]. Obwohl die Anzahl der Schlaganfälle bei Frauen im gebärfähigen Alter insgesamt gering ist, haben Schwangere und Frauen im Wochenbett aufgrund verschiedener Faktoren wie z. B. einer Veränderung der kardiovaskulären Situation oder der Blutgerinnung ein ansteigendes Risiko, einen Schlaganfall zu erleiden [23]. Nach aktueller Datenlage liegt die Inzidenz eines Schlaganfalls bei Schwangeren bzw. in der Postpartalperiode bei 25 bis 34 Fällen pro 100 000, während - im Vergleich dazu - die Inzi- 
denz bei gleichaltrigen Frauen der Normalbevölkerung bei etwa 11 Fällen pro 100000 liegt [24 27]. Während der Gravidität ist das 3. Trimenon vor allem betroffen.

Obwohl nur bei einem kleinen Anteil aller Schwangeren die Diagnose eines Schlaganfalls gestellt wird, sind $12 \%$ aller maternalen Todesfälle auf Schlaganfälle zurückzuführen und gehen zudem mit einer signifikant erhöhten fetalen Morbidität und Mortalität einher. Verschiedene Studien haben gezeigt, dass der arterielle Hypertonus auch bei Schwangeren als wichtiger Risikofaktor zur Entstehung eines Schlaganfalls zu werten ist [24-28]. Die Gründe einer arteriellen Hypertonie in der Schwangerschaft sind unterschiedlich. Ein Hypertonus kann vorbestehend oder durch die Schwangerschaft selbst bedingt sein, und er kann sekundär im Rahmen einer Präeklampsie bzw. Eklampsie auftreten. Als weitere schwangerschaftsassoziierte Risikofaktoren sind Diabetes mellitus, Nikotinabusus und Migräne zu nennen [24, 27].

Kardiogene Hirnembolien treten vor allem bei vorbestehenden Herzklappenerkrankungen, Endokarditiden und Herzrhythmusstörungen auf. Seltenere Ursachen sind Fruchtwasserembolien oder paradoxe Embolien bei offenem Foramen ovale und Unterschenkelvenenthrombose. Die Peripartum-Kardiomyopathie ist eine seltene Schwangerschaftskomplikation mit dem Risiko der Ausbildung intrakardialer Thromben bei einer Ejektionsfraktion $<35 \%$. Sie tritt mit einer Inzidenz von 20 bis 300 pro 100000 Geburten meist innerhalb des letzten Schwangerschaftsmonats bis zu 5 Monate nach Entbindung auf.

Die Gravidität stellt einen relativen hyperkoagulatorischen Zustand dar. Placenta-Trophoblasten produzieren prokoagulatorische Faktoren; zum Zeitpunkt der Placentalösung dient dies der Blutstillung. Fibrinogen ist erhöht, Protein S und C fallen ab. Als Hinweis auf fibrinolytische Vorgänge steigen die D-Dimere im Lauf der Schwangerschaft an.

Das Antiphospholipid-Syndrom geht nicht nur mit Aborten einher, sondern kann bei intakter Schwangerschaft ein HELLP-Syndrom, eine Sinusvenenthrombose oder ischämische Schlaganfälle begünstigen. Auch genetisch determinierte Koagulopathien manifestieren sich klinisch oft erstmals während einer Schwangerschaft durch einen Schlaganfall. Dies gilt insbesondere für die thrombotische thrombozytopenische Purpura (TTP) und die Sichelzellanämie. Komplikationen in der Schwangerschaft, die Wehentätigkeit und die Entbindung per se sowie Hyperemesis gravidarum, Thrombozytopenie oder Infektionen sind ebenfalls mit einem erhöhten Schlaganfallrisiko assoziiert [4].

Schlaganfallursachen, die in direktem Zusammenhang mit der Schwangerschaft bzw. dem Wochenbett stehen, sind die Präeklampsie/Eklampsie, die Sinus- und Venenthrombose und das reversible zerebrale Vasokonstriktionssyndrom (Postpart-
um-Angiopathie, siehe oben). Bei einer Reihe von Vasopathien besteht während der Gravidität ein erhöhtes ischämisches Schlaganfallrisiko, dies gilt beispielsweise für die Moyamoya-Krankheit [29].

$\mathrm{Zu}$ den Therapieoptionen eines akuten ischämischen Schlaganfalls in der Schwangerschaft zählen die intravenöse Lyse mit rekombinantem gewebespezifischem Plasminogenaktivator (rtPA) und die mechanische Thrombektomie. Plazentagängig ist rtPA nicht und in Untersuchungen am Tiermodell ergab sich bisher kein Anhalt für Teratogenität. Nach den aktuellen Zulassungskriterien zählt die Schwangerschaft allerdings als relative Kontraindikation zur Durchführung einer Thrombolyse mit rtPA. Es liegen zwar einige Fallberichte zur erfolgreichen Behandlung während der Schwangerschaft vor, allerdings ist die aktuelle Datenlage ausschließlich auf kleine Fallserien oder Einzelfallberichte beschränkt [30, 31]. Auch wenn es nach diesen so scheint, dass die Thrombolyse während der Schwangerschaft grundsätzlich eine Behandlungsmöglichkeit darstellt, ist dies nach wie vor eine Einzelfallentscheidung und gilt als individueller Heilversuch. Nachdem die Überlegenheit der interventionellen Behandlung gegenüber der i.v.-Lyse inzwischen in mehreren Studien gezeigt wurde [32-35], ist die endovaskuläre Thrombektomie mittels Stent-Retriever die vermutlich beste Therapieoption bei Verschlüssen größerer intrakranieller Arterien, da diese Akutbehandlung ohne Medikamente auskommt. Bislang fehlen allerdings Daten zum Einsatz in der Schwangerschaft und die Strahlenbelastung muss natürlich berücksichtigt werden.

Wichtige Schlaganfallursachen in Schwangerschaft und Wochenbett sind Präeklampsie/ Eklampsie, Sinus- und Venenthrombose und das reversible zerebrale Vasokonstriktionssyndrom (Postpartum-Angiopathie). Veränderungen der kardiovaskulären Situation (Fruchtwasserembolien, Peripartum-Kardiomyopathie) und der Blutgerinnung (TTP, Antiphospholipidsyndrom) sind auszuschließen.

\section{Schwangerschaft und intrakranielle \\ Blutungen}

Die Inzidenz von intrazerebralen (ICB) und subarachnoidalen Blutungen (SAB) ist während der Gravidität erhöht. Hypertonie, Rauchen, Alkoholmissbrauch und Drogenabusus sind bekannte Risikofaktoren. Schwangerschaftsassoziiert spielen die Präeklampsie, ein RCVS, Sinusvenenthrombosen und Gerinnungsstörungen eine Rolle. Aneurysmen, arteriovenöse Malformationen (Angiome) und die Moyamoya-Krankheit können sich unter der Gravidität durch eine Blutung erstmanifestieren [36] und müssen stets ausgeschlossen werden.

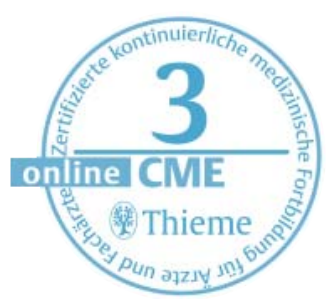




\section{Periphere Nervenläsionen in der \\ Schwangerschaft}

Häufigste periphere neurologische Komplikation der Schwangerschaft ist das Karpaltunnelsyndrom mit einer Inzidenz von 2 bis 35\%. Die vermehrte Flüssigkeitseinlagerung führt zu der typischen Brachialgia nocturna paraesthetica. Wenn die Missempfindungen der ersten 3 Finger volar sich manifestieren, sollte die Diagnose elektroneurografisch abgesichert werden. In der Regel reichen die Ruhigstellung im Handgelenk während der Nachtstunden bzw. Steroidinjektionen lokal aus; nur beim Auftreten motorischer Symptome sollte eine Dekompression erwogen werden [37]. Warum periphere Fazialisparesen während einer Schwangerschaft 2-4 Mal häufiger auftreten, ist unklar. Diskutiert werden perineurale Ödeme, Thromboseneigung der Vasa nervorum und eine relative Immunsuppression. Die Manifestation meist im letzten Trimester oder in der 1 . Woche post partum erlaubt den Einsatz von Kortikoiden ohne Gefährdung des Kindes [38].

Periphere Nervenläsionen der unteren Extremitäten treten bei 58 von 10000 Entbindungen auf [39]. Zu den Nervenläsionen, die sich vor allem postpartal manifestieren, zählen die Meralgia paraesthetica und das Romberg-Howship-Syndrom. Die Läsion des N. cutaneus femoris lateralis kann während der natürlichen Geburt durch die Lagerung der Mutter provoziert werden; die schmerzhaften Parästhesien des ventrolateralen Oberschenkels werden durch Lidocain-Pflaster oder lokale Infiltrationen behandelt

Die Läsion des N. obturatorius kommt zustande, wenn der kindliche Kopf in das Becken eintritt. Es resultieren Missempfindungen an der distalen Innenseite des Oberschenkels (Romberg-HowshipSyndrom) - auch hier helfen lokale Lidocain-Pflaster.

Läsionen anderer Beinnerven (N. femoralis, N. ischiadicus, Plexus lumbosacralis) machen den CToder MR-tomografischen Ausschluss von peripartalen Blutungen (retroperitoneal, M. iliopsoas) erforderlich. Die lumbosakrale Peripartum-Plexopathie tritt sehr selten ante partum in der 32. bis 34 . Gestationswoche mit Rückenschmerzen, Hüftbeugerund Adduktorenschwäche auf. Meist zeigt sie sich intra partum oder post partum bedingt durch Kompression am Beckenrand, und dann auch mit einer Fußheberschwäche, was zur Fehldiagnose eines L5-Syndroms führen kann. Die Inzidenz beträgt etwa 1 auf 2000 bis 6000 Entbindungen [40].

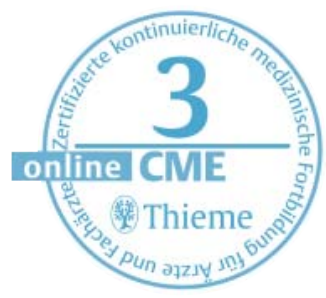

Karpaltunnelsyndrom und periphere Fazialisparesen treten ödembedingt gehäuft in der Schwangerschaft auf. Meralgia paraesthetica und Romberg-Howship-Syndrom durch Kompression post partum.

\section{Extrapyramidale Erkrankungen}

Über 30\% der Frauen entwickeln in der Schwangerschaft ein Restless-Legs-Syndrom (RLS; Willis-Ekbom-Krankheit). In diesen Fällen muss stets ein Eisenmangel als Ursache ausgeschlossen bzw. behandelt werden. Bei ausgeprägten Symptomen kommt therapeutisch die Gabe von Clonazepam infrage. Wenn Verhaltensregeln (Vermeiden langer Ruhephasen, leichtes Ausdauertraining, keine Sedativa oder Serotoninwiederaufnahmehemmer) nicht ausreichend wirken, können jenseits des ersten Trimenons auch L-Dopa oder Oxycodon in möglichst niedriger Dosierung verabreicht werden [41].

Die Chorea gravidarum tritt meist zwischen dem 2. und 5. Schwangerschaftsmonat auf [42]. Gelegentlich haben die Patientinnen zuvor eine SydenhamChorea (minor) bei rheumatischem Fieber durchgemacht. Ein Antiphospholipidsyndrom kann eine Rolle spielen. Ein Morbus Wilson und eine Hyperthyreose müssen laborchemisch ausgeschlossen werden. In aller Regel bessern sich die Hyperkinesen spontan nach der Entbindung. Im Einzelfall können niedrig dosiert Tranquilizer oder Kortikosteroide versucht werden.

Das Restless-Legs-Syndrom (Willis-Ekbom-Krankheit) tritt bei $30 \%$ der Frauen in der Schwangerschaft auf. Stets Eisenmangel ausschließen. Therapie mit Clonazepam und ab dem zweiten Trimenon auch L-Dopa oder Oxycodon.

\section{Schwangerschaft bei neurologischen Erkrankungen Epilepsie}

Patientinnen mit einer vorbekannten Epilepsie haben ein etwa doppeltes Frühgeburt-Risiko. Aufgrund der physiologischen Vorgänge kann es unter der Gravidität infolge einer veränderten Verstoffwechselung der Antikonvulsiva zu einem erhöhten Anfallsrisiko kommen.

Besonders relevant ist das unterschiedlich hohe teratogene Risiko der Substanzen.

Aus diesem Grund sollte eine medikamentöse Umstellung vor geplanter Gravidität mit der Patientin frühzeitig kommuniziert werden.

Kinder, die während der Schwangerschaft gegenüber Antikonvulsiva exponiert waren, haben ein etwa doppeltes Risiko von Geburtsdefekten mit einer Fehlbildungsrate von 4-6\%. Bis zu einem gewissen Grad kann die Gabe von Folsäure vorbeugend nützen, dies gilt insbesondere für enzyminduzierende Substanzen. Grundsätzlich sollte während einer Schwangerschaft nur ein Antikonvulsivum eingesetzt werden - Polytherapien erhöhen das Risiko. Spaltenbildungen im Bereich des Gaumens treten gehäuft unter Diphenylhydantoin und Carbamazepin, kardiale Fehlbildungen unter Phenobarbital auf. 
Das höchste Risiko besteht für Valproinsäure. Zum fetalen Valproinsäure-Syndrom zählen verschiedene kongenitale Malformationen und Dysmorphien. Im Langzeitverlauf können Intelligenzminderungen und autismusähnliche Bilder beim Kind resultieren.

Das geringste Fehlbildungsrisiko weisen Lamotrigin und Levetiracetam auf, sodass nach Möglichkeit vor einer Schwangerschaft auf eine dieser beiden Substanzen umgestellt werden sollte [43]. Grundsätzlich ist die niedrigste wirksame Dosis einer antikonvulsiven Monotherapie vor allem in den ersten 3 Monaten der Gravidität die beste.

Antikonvulsiva während der Schwangerschaft verdoppeln das Risiko von Geburtsdefekten. Das geringste Fehlbildungsrisiko zeigen Lamotrigin und Levetiracetam, das höchste Valproinsäure. Zum fetalen Valproinsäure-Syndrom zählen kongenitale Malformationen, Dysmorphien und im Langzeitverlauf Intelligenzminderungen.

\section{Multiple Sklerose}

Patientinnen mit einer Multiplen Sklerose sollte ein Kinderwunsch nicht verwehrt werden. In der Mehrzahl der Fälle sinkt das Schubrisiko während einer Gravidität; im Wochenbett ist es hingegen erhöht. Ein erhöhtes Risiko für das Kind durch die Schwangerschaft per se besteht nicht.

Nach Möglichkeit sollten Intervalltherapien vor einer geplanten Schwangerschaft beendet werden. Keine der in der MS-Intervalltherapie eingesetzten Substanzen ist während der Schwangerschaft zugelassen. Trotzdem gelten die schon lange eingesetzten injizierbaren Intervalltherapeutika Glatirameracetat und Interferone als relativ sicher. Die dazu vorliegenden Registerdaten zeigen keine erhöhte Rate von Teratogenität oder Fehlgeburten. Immunsuppressive Substanzen bzw. zytostatische Medikamente wie Mitoxantron, Teriflunomid oder Fumarsäure dürfen grundsätzlich während einer Schwangerschaft nicht gegeben werden. Für Fingolimod, Atalizumab und Natalizumab liegen ausreichende Daten nicht vor, so dass auch diese Substanzen strikt vermieden werden müssen [43]. Während im 1. Trimenon die Kortikoidstoßtherapie eines interkurrent auftretenden Schubs nicht unproblematisch ist, gelten entsprechende Behandlungen im 2. und 3. Trimenon als relativ sicher. Sie erhöhen allerdings das Risiko interkurrenter Thrombosen!

Kontrollierte Studien zum Einsatz von intravenösen Immunglobulinen im Wochenbett, um der in dieser Phase erhöhten Schubrate vorzubeugen, liegen nicht vor. Wenn eine Intervalltherapie der Multiplen Sklerose nach der Entbindung zügig wieder aufgenommen werden soll, wird generell empfohlen abzustillen.
Keine der in der MS-Intervalltherapie eingesetzten Substanzen ist während der Schwangerschaft zugelassen, aber Glatirameracetat und Interferone gelten als relativ sicher. Kortikoidstoßtherapie im 2. und 3. Trimenon möglich; intravenöse Immunglobuline im Wochenbett.

\section{Myasthenia gravis}

Bei der Myasthenia gravis werden in der Intervalltherapie klassische Immunsuppressiva wie Azathioprin, Methotrexat oder Mycophenolatmofetil eingesetzt. Diese Substanzen müssen vor einer geplanten Schwangerschaft abgesetzt werden. Die Therapie mit Cholinesterasehemmern kann während der Gravidität fortgeführt werden. Während es bei $1 / 3$ der Patientinnen zu einem geringeren Bedarf während der Schwangerschaft kommt, können bei etwa $20 \%$ Verschlechterungen der Myasthenie auftreten, vor allem im 1 . Trimenon. Noch höher ist das Risiko einer myasthenen Verschlechterung im Wochenbett (etwa 1/3).

$\mathrm{Zu}$ beachten ist, dass Kinder myasthener Mütter maternale Anti-Acetylcholinrezeptor-Antikörper aufweisen und damit eine kongenitale Myasthenie zeigen können. Entsprechend sollten die Vorbereitungen bei der Geburt gemeinsam mit der (Neuro-)Pädiatrie getroffen werden.

Während die glatte Muskulatur durch die Myasthenie nicht betroffen ist, kann die Skelettmuskulatur des Abdomens, die für die natürliche Geburt eingesetzt wird, so beeinträchtigt sein, dass die zusätzliche Gabe von Pyridostigmin unter der Geburt erforderlich ist. In der Regel wird man sich bei protrahierter Geburt zu einer Sectio entscheiden.

Wichtig ist zu bedenken, dass der Einsatz von Magnesiumpräparaten (zur Behandlung einer Präeklampsie) eine Myasthenie bis hin zu einer myasthenen Krise verschlechtern kann!

Risiko einer myasthenen Verschlechterung im Wochenbett und durch Magnesiumpräparate (bei Präeklampsie/Eklampsie). Kongenitale Myasthenie beim Kind durch maternale Anti-Acetylcholinrezeptor-Antikörper.

\section{Hereditäre neuromuskuläre Erkrankungen}

Hereditäre neuromuskuläre Erkrankungen wie die dystrophische Myotonie, die Charcot-MarieTooth-Erkrankung oder spinale Muskelatrophien schließen nicht grundsätzlich eine Schwangerschaft aus. Vor einer geplanten Gravidität sollten die Patientinnen allerdings humangenetisch beraten werden. Bei einer Schwangerschaft ist das Risiko von Frühgeburten erhöht auf etwa 15 bis $30 \%$. Eine Verschlechterung der neurologischen Symptomatik der Mutter infolge der Gravidität ist vor allem bei den Gliedergürtelmuskeldystrophien möglich.

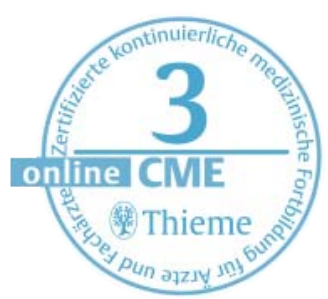




\section{Kopfschmerzen}

Bei der Mehrzahl aller Schwangeren mit primären Kopfschmerzen (Spannungskopfschmerz, Migräne) ist die Diagnose vorbekannt; nur in 10\% kommt es zur Erstmanifestation während der Gravidität [44]. In diesen Fällen handelt es sich bei einem Drittel um eine Migräne und bei einem weiteren Drittel um präeklampsie-, eklampsiebedingte Kopfschmerzen. Besondere Vorsicht ist geboten, wenn sich atypische Kopfschmerzen während der Gravidität erstmanifestieren. In diesen Fällen müssen stets ein reversibles Vasokonstriktionssyndrom (RCVS), ein posteriores reversibles Enzephalopathie-Syndrom (PRES) und eine Sinusvenenthrombose ausgeschlossen werden. Des Weiteren ist an die Dissektion zerebraler $\mathrm{Ar}$ terien, infektiöse Ursachen und die zerebrale autosomal dominante Arteriopathie mit subkortikalen Infarkten und Leukenzephalopathie (CADASIL) zu denken, die sich während einer Schwangerschaft erstmals zeigen kann.

Grundsätzlich sollten Patientinnen mit primären Kopfschmerzformen eine etwaige Intervalltherapie vor geplanter Schwangerschaft beenden. Nach den vorliegenden Daten führen die hormonellen Veränderungen während der Gravidität in der Regel zu einem deutlichen Rückgang der Frequenz einer Migräne [etwa $80 \%$ ]. In der 1 . Woche post partum tritt die Migräne bei $34 \%$ und im 1. Monat nach Entbindung bei 55\% wieder auf [45]. Wenn die Mutter stillt, hält der positive Effekt der Hormonumstellung bis zu 6 Monate nach der Entbindung an. Allerdings erhöht das Vorliegen einer Migräne in der Vorgeschichte das Risiko einer Hypertonie und von Schlaganfällen während der Schwangerschaft und im Wochenbett.

Im Unterschied zur Migräne ändert sich die Häufigkeit eines Kopfschmerzes vom Spannungstyp durch die Schwangerschaft nicht. Bei beiden Kopfschmerzformen können Acetaminophen und Koffein im Bedarfsfall während der Gravidität am ehesten verabreicht werden.

Beim Postpartumkopfschmerz handelt es sich am häufigsten um muskuläre oder Spannungskopfschmerzen, die durch Schlafstörungen, unregelmäßiges Essen und Stress begünstigt werden [46]. Lageabhängige Kopfschmerzen im Wochenbett treten vor allem nach einer Periduralanästhesie zur Entbindung auf (Liquorunterdrucksyndrom) und können das Auftreten einer Sinusvenenthrombose begünstigen [47].

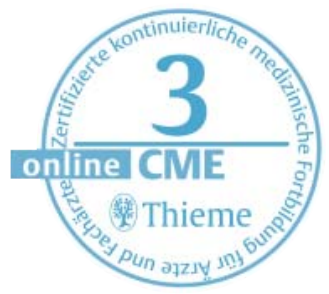

Während der Gravidität meist deutlicher Rückgang der Migränefrequenz. Bei atypischen Kopfschmerzen stets reversibles Vasokonstriktionssyndrom, Dissektionen, ein posteriores reversibles Enzephalopathie-Syndrom und eine Sinusvenenthrombose ausschließen! Post partum meist Spannungskopfschmerzen.
Interessenkonflikt: Der Autor gibt an, dass kein Interessenkonflikt besteht.

\section{Literatur}

1 Cnossen JS, Vollebregt KC, de Vrieze $N$ et al. Accuracy of mean arterialpressure and blood pressure measurements in predicting preeclampsia:systematic review and meta-analysis. BMJ 2008; 336: 1117-1120

2 Brewer J, Owens MY, Wallace K et al. Posterior reversible encephalopathy syndrome in 46 of 47 patients with eclampsia. Am J Obstet Gynecol 2013; 208: 468

3 Fletcher JJ, Kramer AH, Bleck TP et al. Overlapping features of eclampsia and postpartum angiopathy. Neurocrit care 2009; 11: 199-209

4 Crovetto F, Somigliana E, Peguero A et al. Stroke during pregnancy and preeclampsia. Current opinion in obstetrics \& gynecology 2013; 25: 425 - 432

5 Sawle GV, Ramsay MM. The neurology of pregnancy. J Neurol Neurosurg and Psychiatry 1998; 64: 711 725

6 [Anonym]. Which anticonvulsant for women with eclampsia? Evidence from the Collaborative Eclampsia Trial. Lancet 1995; 345: 1455-1463

7 Fugate JE, Ameriso SF, Ortiz G et al. Variable presentations of postpartum angiopathy. Stroke 2012; 43: 670-676

8 Call GK, Fleming MC, Sealfon $S$ et al. Reversible cerebral segmental vasoconstriction. Stroke 1988; 19: $1159-1170$

9 Raps EC, Galetta SL, Broderick $M$ et al. Delayed peripartum vasculopathy: cerebral eclampsia revisited. Ann Neurol 1993; 33: 222-225

10 Fugate JE et al. Fulminant postpartum cerebral vasoconstriction syndrome. Arch Neurol 2012; 6: 1111 1117

11 Singhal AB, Bernstein RA. Postpartum angiopathy and other cerebral vasoconstriction syndromes. Neurocrit Care 2005; 3: 91 - 97

12 Krämer M, Berlit P. Reversibles zerebrales Vasokonstriktionssyndrom vs. zerebrale Vaskulitis. Nervenarzt 2012; 82: 500-505

13 Chen SP, Fuh JL, Wang SJ. Reversible cerebral vasoconstriction syndrome: an under-recognized clinical emergency. Ther Adv Neurol Disord 2010; 3: 161 171

14 Kramer MS, Rouleau J, Baskett TF et al. Amniotic-fluid embolism and medical induction of labour: a retrospective, population-based cohort study. Lancet 2006; 368: 1444 - 1448

15 Sipes SL, Malee MP. Endocrine disorders in pregnancy. Obstet Gynecol Clin North Am 1992; 19: 655677

16 Harbeck B, Schutt M, Sayk F. Endocrine emergencies during pregnancy. Med Klin Intensivmed Notfallmed 2012; 107: 110-117

17 Lanska DJ, Kryscio RJ. Risk factors for peripartum and postpartum stroke and intracranial venous thrombosis. Stroke 2000; 31: 1274-1282

18 Lanska DJ, Kryscio RJ. Stroke and intracranial venous thrombosis during pregnancy and puerperium. Neurology 1998; 51: $1622-1628$

19 Canhao P, Ferro JM, Lindgren AG et al. Causes and predictors of death in cerebral venous thrombosis. Stroke 2005; 36: 1720-1725

20 Einhäupl K, Stam J, Bousser MG et al. EFNS guideline on the treatment of cerebral venous and sinus thrombosis in adult patients. Eur J Neurol 2010; 17: $1229-1235$

21 Go AS, Mozaffarian D, Roger VL et al. Heart disease and stroke statistics-2013 update: a report from the 
American Heart Association. Circulation 2013; 127 : e6-e245

22 Seshadri S, Beiser A, Kelly-Hayes $M$ et al. The lifetime risk of stroke: estimates from the Framingham Study. Stroke 2006; 37: 345 - 350

23 Lamy C, Sharshar T, Mas JL. Cerebrovascular diseases in pregnancy and puerperium. Revue neurologique 1996; 152: $422-440$

24 James $A H$, Bushnell $C D$, Jamison MG et al. Incidence and risk factors for stroke in pregnancy and the puerperium. Obstet Gynecol 2005; 106: 509-516

25 Kittner SJ, Stern BJ, Feeser BR et al. Pregnancy and the risk of stroke. N Eng J Med 1996; 335: 768-774

26 Berlit $P$, Schönenberger S. Schwangerschaft und Schlaganfall. Akt Neurol 2014; 41: 443-446

27 Sharshar T, Lamy C, Mas JL. Incidence and causes of strokes associated with pregnancy and puerperium. A study in public hospitals of Ile de France. Stroke in Pregnancy Study Group. Stroke 1995; 26: 930-936

28 Jaigobin C, Silver FL. Stroke and pregnancy. Stroke 2000; 31: $2948-2951$

29 Krämer M, Berlit P. Die Moyamoya-Erkrankung - Eine seltene Vasopathie bei europäischen Patienten. Fortschritte Neurol Psychiatr 2010; 78: 542-553

30 Leonhardt G, Gaul C, Nietsch HH et al. Thrombolytic therapy in pregnancy. J Thromb Thrombolysis 2006; 21: $271-276$

31 Broderick JP. Should intravenous thrombolysis be considered the first option in pregnant women? Stroke 2013; 44: 866-867

32 Saver JL, Goyal M, Bonafe A et al. Stent-retriever thrombectomy after intravenous t-PA vs. t-PA alone in stroke. N Engl J Med. DOI: 10.1056/NEJMoa1415061

33 Goyal M, Demchuk AM, Menon BK et al. Randomized assessment of rapid endovascular treatment of ischemic stroke. N Engl J Med 2015; 372: 1019-1030

34 Campbell BC, Mitchell PJ, Kleinig TJ et al. Endovascular therapy for ischemic stroke with perfusion-imaging selection. N Engl J Med 2015; 372: 1009-1018
35 Berkhemer OA, Fransen PS, Beumer D et al. A randomized trial of intraarterial treatment for acute ischemic stroke. N Engl J Med 2015; 372: 11 - 20

36 Bateman BT, Schumacher HC, Bushnell CD et al. Intracerebral hemorrhage in pregnancy: frequency, risk factors, and outcome. Neurology 2006; 67: 424-429

37 Padua L, Aprile I, Caliandro $P$ et al. Symptoms and neurophysiological picture of carpal tunnel syndrome in pregnancy. Clin Neurophysiol 2001; 112: 1946

38 Cohen Y, Lavie O, Granovsky-Grisaru S et al. Bell palsy complicating pregnancy: a review. Obstet Gynecol Surv 2000; 55: 184

39 Wong CA, Scavone BM, Dugan S et al. Incidence of postpartum lumbosacral spine and lower extremity nerve injuries. Obstet Gynecol 2003; 101: 279

40 Feasby TE, Burton SR, Hahn AF. Obstetrical lumbosacral plexus injury. Muscle Nerve 1992; 15: 937

41 Neyal A et al. A prospective study of Willis-Ekbom disease/restless legs syndrome during and after pregnancy. Sleep Med 2015; 16: 1036-1040

42 Cardoso F. Chorea gravidarum. Arch Neurol 2002; 59: 868

43 Berlit P. Memorix Neurologie; 2016, (im Druck)

44 MacGregor EA. Headache in pregnancy. Neurol Clin 2012; 30: 835

45 Sances G, Granella F, Nappi RE et al. Course of migraine during pregnancy and postpartum: a prospective study. Cephalalgia 2003; 23: 197

46 Goldszmidt E, Kern R, Chaput A et al. The incidence and etiology of postpartum headaches: a prospective cohort study. Can J Anaesth 2005; 52: 971

47 Kraayvanger L, Berlit P. Postpartale sekundäre Sinusvenenthrombose mit axialer transtentorieller und tonsillärer Herniation als seltene Komplikation eines Liquorunterdrucksyndroms nach spinaler Anästhesie. Fortschr Neurol Psychiatr 2014; 82: 1 -3

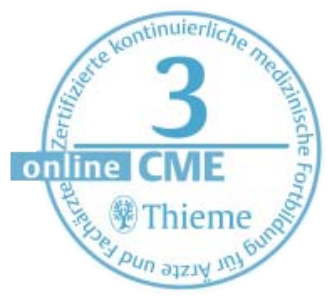




\section{CME-Fragen Neurologische Erkrankungen und Schwangerschaft - eine Übersicht}

1 Welcher der folgenden Befunde zählt zu den diagnostischen Kriterien der Präeklampsie?

Kopfschmerzen

Sehstörungen

Proteinurie

Epilepsie

Knöchelödeme

Bei der sogenannten Postpartum-Angiopathie handelt es sich in erster Linie um ein posteriores reversibles Enzephalopathie-Syndrom ein reversibles zerebrales Vasokonstriktions-Syndrom ein HELLP-Syndrom ein Fruchtwasser-Emboliesyndrom ein Sheehan-Syndrom

Das zerebrale Vasokonstriktions-Syndrom kann durch Medikamente ausgelöst werden. Zu den möglicherweise auslösenden Substanzen zählen am wenigsten wahrscheinlich:

A Serotoninwiederaufnahmehemmer

B Triptane

C abschwellende Nasensprays

D Erythrozytenkonzentrate

E Kalziumantagonisten

Zum Nachweis einer Sinusvenenthrombose in der Schwangerschaft ist in erster Linie geeignet: die zerebrale Angiografie

B die Phasenkontrastdarstellung in der Magnetresonanztomografie

C der Nachweis des Empty triangle-Zeichens in der kontrastmittelgestützten Computertomografie

D der Nachweis erhöhter D-Dimere

E die kontrastmittelgestützte farbcodierte Duplexsonografie

Bei der 25-jährigen Primapara ist es nach der natürlichen Geburt zu einer atonen Nachblutung gekommen, welche die Gabe von insgesamt 9 Bluttransfusionen erforderlich gemacht hat. Wegen deutlich erniedrigter Blutdruckwerte musste die Patientin über 3 Tage intensivmedizinisch überwacht werden. Am 3. Tag post partum entwickelt sie Kopfschmerzen und Sehstörungen. Trotz Besserung der Blutwerte und Volumensubstitution persistieren deutlich erniedrigte Blutdruckwerte (systolisch unter 90). Welche Diagnose ist in erster Linie zu stellen?

A Apoplex der Hypophyse

B Sinusvenenthrombose

C postpartale Angiopathie

D posteriores Enzephalopathie-Syndrom

E HELLP-Syndrom
6 Welches der folgenden Antikonvulsiva kann bei Einnahme während der Schwangerschaft zu einer Intelligenzminderung und zum Autismus beim Kind führen?

A Carbamazepin

B Diphenylhydantoin

C Levetiracetam

D Valproinsäure

E Lamotrigin

7 Was wird unter einem Romberg-Howship-Syndrom verstanden?

A Nächtliche Schmerzen mit Kribbelparästhesien im Bereich der Hände

B Schmerzen und Missempfindungen an der distalen Oberschenkelinnenseite

c Eine schmerzhafte Hyperpathie an der Oberschenkelaußenseite

D Ziehende Schmerzen vom Rücken zur großen Zehe mit Taubheitsgefühl am Fußrücken

E Gürtelförmige Schmerzen und Parästhesien im Abdominalbereich

8 Welcher Nerv ist beim Romberg-Howship-Syndrom betroffen?

N. ischiadicus

N. femoralis

N. cutaneus femoris lateralis

N. saphenus

E N. obturatorius

Welche Aussage zum Verlauf neurologischer Erkrankungen während der Schwangerschaft trifft nicht zu?

A Die Häufigkeit von Migräneattacken nimmt während der Schwangerschaft $a b$

B Bei Gliedergürtelmuskeldystrophien verbessert sich die muskuläre Kraft während der Gravidität

C Die Wahrscheinlichkeit akuter Schübe bei der Multiplen Sklerose nimmt während der Schwangerschaft ab

D Das Risiko epileptischer Anfälle nimmt bei gleichbleibender Dosierung des Antikonvulsivums während der Gravidität zu

E Verschlechterungen einer Myasthenie treten vor allem im ersten Trimenon auf 
10 Welche Aussage zur Therapie neurologischer Komplikationen während der Schwangerschaft trifft nicht zu?

A Beim embolischen Verschluss intrakranieller Arterien ist die interventionelle Behandlung mittels Stent-Retriever eine gute Therapieoption

B Bei der Eklampsie sollte Carbamazepin zur Anfallstherapie eingesetzt werden

C Ein akuter Schub bei Multipler Sklerose im dritten Trimenon wird mittels Kortikoid-Stoßtherapie behandelt

D Auch beim Nachweis von intrazerebralen Blutungen wird die Sinusvenenthrombose antikoaguliert

E Eine Myasthenie wird während der Schwangerschaft mit Cholinesterasehemmern behandelt 